\title{
Erfolgreich berichten
}

Deutsche Manager agieren nicht selten auf Basis unzureichender oder ungeeigneter Daten. Nur ein Viertel aller Führungskräfte gibt an, dass sie erstens vollständige, zweitens relevante Berichte erhalten. Dies und mehr zeigt die Studie „Unternehmenssteuerung 2016“ der Unternehmensberatung Kampmann, Berg \& Partner (KB\&P). Mehr als 250 Führungskräfte wurden dafür befragt. Weitsichtige Entscheidungen, die auf fundierten Informationen gründen - in vielen deutschen Unternehmen ist das reine Theorie. Manager der höheren Führungsebenen erhalten so viele Berichte, dass mehr als die Hälfte keine Zeit finden, diese überhaupt zu lesen. Das liegt auch daran, dass die Berichte oft nicht brauchbar sind: 59 Prozent halten die Daten für unvollständig und 57 Prozent beklagen irrelevante Reports. Nur 26 Prozent erhalten Berichte, die vollständige und relevante Informationen liefern.

Um die entscheidenden Hebel in der Unternehmenssteuerung zu identifizieren, hat KB\&P auf Basis der Studienergeb- nisse einen Performance-Score entwickelt. Auf dessen Grundlage zeigen sich deutliche Unterschiede zwischen Unternehmen mit einer ergebnisorientierten Führungskultur und jenen, bei denen die Zielerreichung einen geringeren Stellenwert einnimmt. Die erfolgreichsten Unternehmen reporten demnach nicht mehr oder weniger als die Low-Performer. „Aber sie machen es besser - konsequent und mit hoher Aussagekraft", sagt Dr. Torge Doser, Executive Partner bei KB\&P. Ein ähnliches Bild zeigt sich auch in der Planung. Bei knapp 50 Prozent der Unternehmen führen Planverfehlungen zu keinen persönlichen Konsequenzen, und knapp ein Drittel leiten zudem auch keine Maßnahmen für die Zukunft ab, um diese zu vermeiden.

Gabi Böttcher

Weitere Informationen zum Thema gibt es unter www.kampmann-berg.de.

\section{Trotz niedrigem Basiszinssatz Risikozuschlag für Investitionen gestiegen}

Der durchschnittliche Basiszinssatz hat mit 1,5 Prozent in Deutschland und Österreich und 1,3 Prozent in der Schweiz erneut einen historischen Tiefststand erreicht. Das zeigt die Kapitalmarktstudie 2016 der Beratungsgesellschaft KPMG. Dafür wurden rund 200 Unternehmen aus Deutschland, Österreich und der Schweiz befragt. Bei den Umfrageteilnehmern handelte es sich um 77 Prozent der im DAX sowie 46 Prozent der im M-DAX notierten Unternehmen.

Obwohl der risikolose Basiszinssatz gesunken ist, zeigen die Ergebnisse jedoch, dass viele Firmen nach wie vor von hohen Renditeforderungen seitens der Investoren ausgehen. Eine Investition macht für viele nur dann Sinn, wenn die zu erwartenden Erträge über den Kosten für das eingesetzte Kapital liegen. Die angesetzten Kapitalkosten (WACC) sind jedoch nicht gesunken und liegen der Studie zufolge immer noch bei 7,1 Prozent. Das liegt daran, dass der von Investoren geforderte Risikozuschlag für Investitionen in Unternehmen auf 6,4 Prozent in Deutschland gestiegen ist. Die Fremdkapitalkosten betragen aufgrund höherer Risikoaufschläge durch- schnittlich 3,4 Prozent und sind damit, trotz Niedrigzinspolitik, unverändert im Vergleich zum Vorjahr. Branchenbezogen zeigen sich aber interessante Unterschiede. Die Renditeforderungen der Kapitalgeber sind branchenübergreifend nahezu konstant. Bei den Kapitalkosten gaben hingegen die teilnehmenden Unternehmen aus den Bereichen Technology (7,9 Prozent), Automotive und Industrial Manufacturing (jeweils 7,6 Prozent) die höchsten Werte an. Unternehmen aus den Bereichen Energy \& Natural Resources (6,3 Prozent), Health Care und Transport \& Leisure (jeweils 6,9 Prozent) weisen dagegen die niedrigsten Kapitalkosten aus. Im Vergleich zum Vorjahr sind die Renditeforderungen für die Bereiche Health Care (1,2 Prozent) und Technology (1,1 Prozent) gestiegen, während sie im Bereich Media \& Telecommunications um 0,8 Prozentpunkte gesunken sind.

Sylvia Meier

Weitere Details finden Sie unter https://home.kpmg.com/ de/de/home/themen/2016/11/kapitalkostenstudie-2016.html. 